Article

\title{
Performance Analysis of Bandwidth Allocation Based on Dynamic User Priority for Indoor Visible Light Communication Ultra-Dense Networks
}

\author{
Xiang-Wei Bai ${ }^{\circledR}$, Qing $\mathrm{Li}^{*}$ and Si-Yu Tao \\ National Digital Switching System Engineering and Technological Research Center (NDSC), \\ Zhengzhou 450001, China; bai940102@163.com (X.-W.B.); peachforworking@foxmail.com (S.-Y.T.) \\ * Correspondence: liqing0206@163.com; Tel.: +86-0371-81624122
}

Received: 2 January 2019; Accepted: 22 February 2019; Published: 2 March 2019

check for updates

\begin{abstract}
Focusing on the high user density in visible light communication ultra-dense networks (VLC-UDNs), this paper proposes a resource allocation method based on dynamic user priority (DUP). Firstly, this paper establishes the DUP model, which realizes a multi-dimensional measurement for differences of users. Considering the variety of network environments, we dynamically select multiple features of users and achieve the calculation of DUP by fuzzy logic (FL). Secondly, the throughput-maximizing resource allocation (TMRA) scheme with user priority guarantee is proposed. Thirdly, the lower bound of the proposed DUP-TMRA is derived. Simulation results show that the proposed multi-dimensional DUP model outperforms the conventional one-dimensional DUP model and fixed priority model. In addition, the proposed TMRA scheme outperforms the conventional proportion allocation scheme. Finally, in comparisons of system throughput, the proposed DUP-TMRA achieves $4 \%$ performance improvement against the conventional required data rate proportion allocation (RPA) method. In comparisons of fairness, DUP achieves the modest performance. In comparisons of satisfaction, when the average blocking probability is higher than 0.45 , the proposed DUP-TMRA improves the proportion of satisfied users against the conventional RPA method by up to $17.5 \%$.
\end{abstract}

Keywords: visible light communication networks; resource allocation; dynamic user priority; fuzzy logic

\section{Introduction}

Visible light communication (VLC) is an emerging wireless communication technology with advantages of large bandwidth and high speed [1]. Due to the ubiquitous indoor light sources, VLC receives the extensive attention from both the academic community and the industry, and it becomes an attractive alternative to conventional radio communications for indoor environments [2,3].

According to the number of light sources and terminals, the indoor VLC networks include a low-density scene and a high-density scene. In a low-density scene represented by the home environment, the number of light sources and the number of terminals is small, while the number of terminals is slightly greater than that of light sources. In contrast, in a high-density scene represented by large-scale indoor waiting rooms, the number of light sources and terminals is large and the number of terminals is much greater than that of light sources. Compared with the typical parameters of radio frequency ultra-dense networks (RF-UDNs) [4], the parameters of VLC networks in a high-density scene have reached the critical values of the relevant parameters in RF-UDNs. For example, the traffic demand in indoor communication area has reached $10 \mathrm{Mbps} / \mathrm{m}^{2}$ [4]. Due to the high user density, high access point (AP) and high traffic density requirement, the indoor VLC networks should provide 
high-density and high-rate services. We refer the VLC networks with above three high-density features as visible light communication ultra-dense networks (VLC-UDNs).

Different from the radio signals in traditional RF-UDNs, the visible light in VLC-UDNs has a line of sight (LOS) and is easily to be blocked to cause communication interruption. On one hand, the high user density makes the interruption more frequent. On the other hand, the strong directivity of light sources makes the coverage area of a single light source limited $[5,6]$ and the user channel quality is closely related to user's spatial location. Therefore, in the condition of high traffic density and diversified traffic types, the resource allocation (RA) problem of VLC-UDNs is a key issue [7].

Focusing on the high user density, this paper study the throughput-maximizing RA problem based on dynamic user priority (DUP) in single-cell VLC-UDNs. [8-10] used the Markov chain model to assign visible multi-color sub-channels, measured user priority based on the types of traffic, and provided better services to the users with higher priority. Using the concept of channel reservation, [11] proposed an adaptive bandwidth allocation method and measured user priority based on the types of traffic. By releasing bandwidth from existing lower priority users to accept higher priority users, [11] improved the number of served users. The user priority based on traffic type in [8-11] cannot fully reflect the difference among users and the dynamic nature of the network. Based on the orthogonal frequency-division multiplexing (OFDM) system, [12] proposed a three-state fuzzy logic (FL) method for sub-carrier and power resource allocation. Compared with the traditional two-state algorithm, both throughput and user satisfaction performance were improved. Reference [13] proposed the bidirectional allocation game (BAG) algorithm which measured the priority based on fuzzy logic and allocated time slots to users in descending order of priority. In order to offer a proportional fairness among users, [14] allocated time resource based on the required data rate of users. Reference [15] allocated time resource based on the degree of satisfaction of users and improved the performance of both system throughput and user fairness. References [12-15] proposed a more comprehensive user priority evaluation index for resource allocation in dynamic networks. Through the analysis of the above literatures, we conclude that resource allocation involves two key issues, one is the user priority measurement and the other is the optimal resource allocation.

In this paper, a resource allocation method based on DUP is proposed which focuses on high user density for indoor VLC-UDNs. The implementation process of the proposed resource allocation algorithm consists of three steps. The first step is the selection of user's features. Three features are selected dynamically in real time to reflect the difference among users. They are user's channel gain, required data rate, and blocking probability. The second step is the calculation of user's priority and it is achieved with fuzzy logic method which consists of fuzzification, rule evaluation, and defuzzification. The input parameters of the fuzzy logic system are the selected three features and the output is a priority value which is used to guide the resource allocation process. The third step is the achievement of resource allocation which is formulated as an optimization model and a throughput-maximizing resource allocation (TMRA) scheme with priority constraints is proposed. The optimization model could be solved by classical optimization algorithm, such as inter-point method which has a low complexity. Based on the DUP model and the TMRA scheme, the resource allocation algorithm DUP-TMRA is proposed. The simulation results show that the proposed resource allocation method improves the system throughput against the conventional required data rate proportion allocation (RPA) method by $4 \%$. In addition, when the average blocking probability is higher than 0.45 , it improves the proportion of satisfied users by up to $17.5 \%$.

The remainder of this paper is organized as follows. The problem analysis is described in Section 2. The resource allocation method based on DUP is proposed in Section 3. Simulation results are presented in Section 4. Finally, the conclusions are given in Section 5.

\section{Problem Analysis}

As shown in Figure 1, the single-cell VLC-UDNs consist of one access point (AP) and N users. It is assumed that the users are uniformly distributed on the room floor and the positions of users are fixed. 
The users use the photo diode (PD) as a receiver. $\Phi_{1 / 2}$ is the radiation angle at which the intensity is half of the intensity at the main-beam direction; $\phi_{k}$ is the angle of irradiance of the $k$-th user; $\psi_{k}$ is the angle of incidence of the $k$-th user; $\Psi_{c}$ is the field of view (FOV) of users; $h$ is the vertical distance between $\mathrm{AP}$ and a certain receiver; $d_{k}$ is the distance between $\mathrm{AP}$ and the receiver of the $k$-th user.

Classical literature often selects a certain traffic attribute such as the required data rate to reflect the difference among users. In VLC-UDNs, except for traffic attributes, the environmental factors such as the position of the users can also result in difference on resource utilization efficiency. Therefore, a single feature cannot reflect the difference among users effectively. In this paper, the proposed user priority measurement model can be regarded as a mapping of multi-dimensional user feature vector to a priority value. Priority value $Q=F(V)=F\left(v_{1}, v_{2}, \cdots, v_{n}\right)$, where $F$ is the mapping function; $V=\left(v_{1}, v_{2}, \cdots, v_{n}\right)$ is a multi-dimensional user feature vector. Therefore, the key issues are the selection of effective features and the establishment of mapping function. Aiming at the single-cell VLC-UDNs, this paper selects three features to reflect the difference among users and establishes the user dynamic priority model through FL.

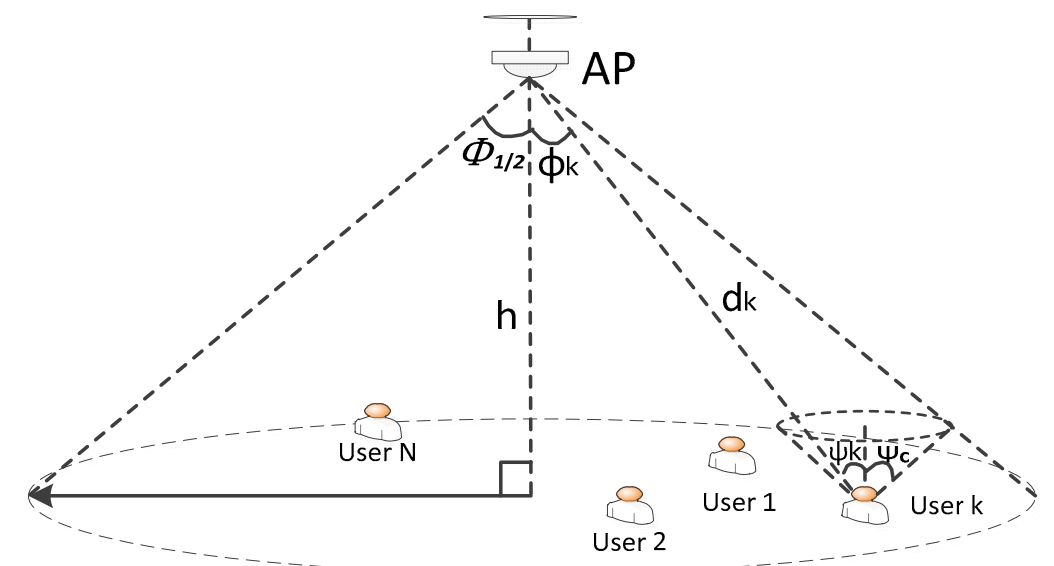

Figure 1. The system model of optical single-cell visible light communication ultra-dense networks (VLC-UDNs).

It is assumed that the single cell is based on OFDM system and the power of each optical channel is uniform. Therefore, the resource allocation problem equals to assigning sub-bands in nature. Assuming that the total channel bandwidth is $B$, the key problem is to seek a division of $B$ to maximize the system throughput under priority constraints.

\section{Resource Allocation Method Based on Dynamic User Priority}

\subsection{Outline}

The model design of the proposed resource allocation method based on DUP is shown in Figure 2. Firstly, this paper selects user's position information, blocking information and traffic information for the measurement of user priority. The position of a user has an impact on channel quality; the blocking information reflects the high density of users and the influence among users; the traffic information reflects the difference on traffic requirement among users. Therefore, the selected three features can reflect the difference among users effectively and be used to measure priority in multiple angles.

Secondly, FL is selected as the user priority mapping function to calculate user priority due to its multi-dimensional feature decision ability and comprehensive measurement ability. Using users' information periodically, the priority of users is dynamically changed to adapt to dynamic network environment. Finally, bandwidth allocation is achieved through resource allocation unit. 


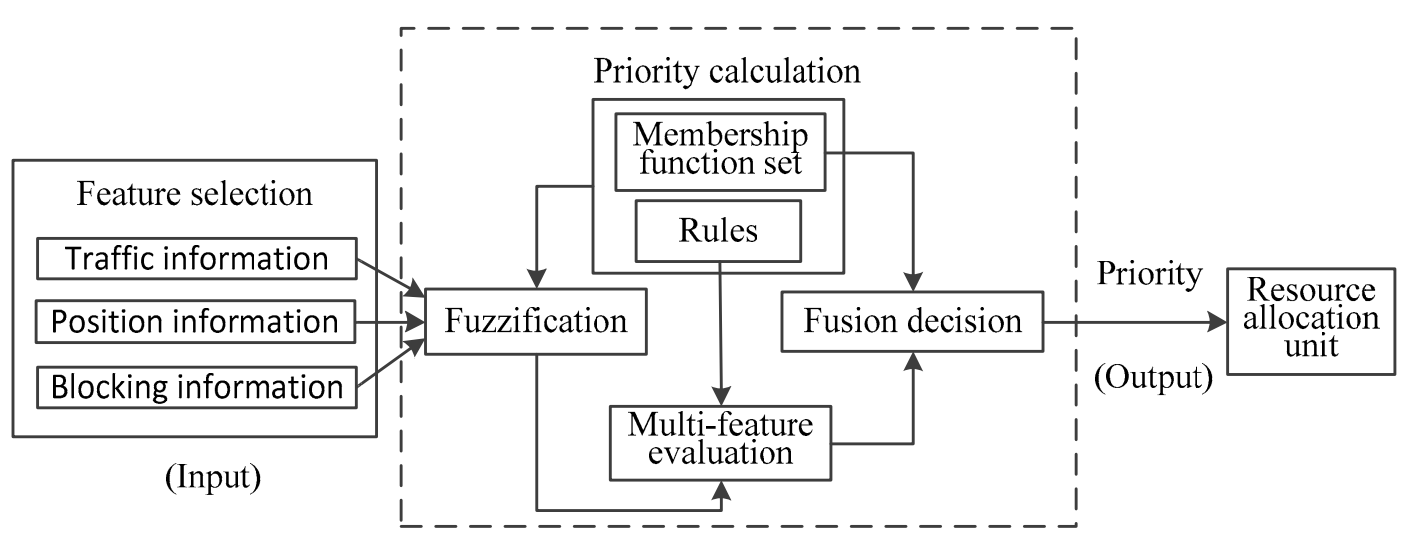

Figure 2. Model design.

\subsection{Dynamic User Priority}

\subsubsection{User Feature Selection}

Three features are selected to reflect the difference among users. They are channel gain, blocking probability, and required data rate and they represent user's position information, blocking information, and traffic information, respectively.

- Channel gain

The channel gain of the $k$-th user is given by [16]:

$$
H_{k}=\left\{\begin{array}{l}
\frac{(m+1) A}{2 \pi d_{k}^{2}} \cos ^{m}\left(\phi_{k}\right) T_{S}\left(\psi_{k}\right) g\left(\psi_{k}\right) \cos \left(\psi_{k}\right), \quad 0 \leq \psi_{k} \leq \Psi_{c}, \\
0, \quad \psi_{k}>\Psi_{c}
\end{array}\right.
$$

where $m=-\ln 2 /\left(\ln \left(\cos \Phi_{1 / 2}\right)\right)$ is the Lambertian emission order; $A$ is the physical area of PD; $T_{S}(\psi)$ is the gain of the optical filter; $g\left(\psi_{k}\right)$ denotes the optical concentrator gain, which is formulated as

$$
g\left(\psi_{k}\right)=\left\{\begin{array}{cl}
\frac{n^{2}}{\sin ^{2} \Psi_{c}}, & 0 \leq \psi_{k} \leq \Psi_{c} \\
0, & \psi_{k}>\Psi_{c}
\end{array}\right.
$$

where $n$ is the refractive index. For the $k$-th user, the signal to noise ratio (SNR) is given by [17]

$$
\gamma_{k}=\frac{\left(r P_{k} H_{k}\right)^{2}}{n_{0} B_{k}}
$$

where $r$ is the responsivity of PDs; $P_{k}$ is the received optical signal power of the $k$-th user; $n_{0}$ is the power spectral density of noise; $B_{k}$ is the bandwidth allocated to the $k$-th user.

- Blocking probability

Due to the obstruction, the LOS link of the $k$-th user might be blocked. It is assumed that the blocking event random variable obeys the Bernoulli distribution and its probability mass function may be given by [18]

$$
I\left(\kappa_{k}\right)= \begin{cases}1-p_{k}, \kappa_{k}=1 \\ p_{k}, & \kappa_{k}=0\end{cases}
$$

where $p_{k}$ denotes the blocking probability of the $k$-th user. In order to reflect the difference of the channel quality among users, we assume that $p_{k}$ obeys the Beta distribution. The achievable data rate between the $k$-th user and AP is expressed as [19]

$$
C_{k}=\left(1-p_{k}\right) B_{k} \log _{2}\left(1+\gamma_{k}\right)
$$

- Required data rate 
The required data rate of the $k$-th user, denoted by $R_{k}$, obeys to a Gamma distribution [14]. In a time period, we assume that the position, blocking probability, and required data rate of each user are independently and identically distributed. The mathematical expectations of user's blocking probability and required data rate are denoted by $\bar{p}$ and $\bar{R}$, respectively.

\subsubsection{User Priority Calculation}

The FL system generates a weight value dynamically for a user as a priority according to the user's channel gain $H$, blocking probability $p$ and required data rate $R$.

- Fuzzification

Each user feature is classified into several categories that are used to reflect the level difference of a certain user feature. Each category has a membership function (MF). When the value of user feature is determined, it could be substituted into different MFs to calculate the membership degrees to different categories. Fuzzification is the first step of FL, which converts each user feature value into the membership degrees to different categories. There is a wide selection of MFs and the only condition an MF must satisfy is that it must range from 0 to 1 . We adopt a trapezoidal-shaped function as an MF, which is a generalization of triangle-shaped function [14]

$$
f(x ; a, b, c, d)=\left\{\begin{array}{cc}
0, & x \leq a \\
\frac{x-a}{b-a}, & a \leq x \leq b \\
1, & b \leq x \leq c \\
\frac{d-x}{d-c}, & c \leq x \leq d \\
0, & x \geq d
\end{array}\right.
$$

where coefficients $a, b, c$, and $d$ for each category are different, but normally need to provide overlap between the MFs of two neighbouring categories. For a certain category, $a$ is the minimum value of the category and $d$ is the maximum value of the category. The sets of MFs for $R, H$, and $p$ are expressed as (7), (8), and (9), respectively

$$
\begin{aligned}
& F_{R}\left(n_{1}\right)=\left\{f_{i}^{R}\left(R ; v_{i}^{R}=\left(a_{i}, b_{i}, c_{i}, d_{i}\right)\right) \mid i=1, \cdots, n_{1}\right\}, \\
& F_{H}\left(n_{2}\right)=\left\{f_{i}^{H}\left(H ; v_{i}^{H}=\left(a_{i}, b_{i}, c_{i}, d_{i}\right)\right) \mid i=1, \cdots, n_{2}\right\}, \\
& F_{p}\left(n_{3}\right)=\left\{f_{i}^{p}\left(p ; v_{i}^{p}=\left(a_{i}, b_{i}, c_{i}, d_{i}\right)\right) \mid i=1, \cdots, n_{3}\right\} .
\end{aligned}
$$

Equation (7) represents that user feature $R$ is classified into $n_{1}$ categories and it has $n_{1}$ MFs accordingly. Similarly, feature $H$ has $n_{2}$ MFs and feature $p$ has $n_{3}$ MFs. The values of $n_{1}, n_{2}$ and $n_{3}$ are at least 2 and they reflect the precision of level difference for a category.

We choose the channel gain as an example to describe the fuzzification process as shown in Figure 3. When $n_{2}=3$, the channel gain is classified into three categories: 'low', 'medium', and 'high', and corresponding parameters are $v_{1}^{H}=(0,0,1.0,1.4) \times 10^{-5}, v_{2}^{H}=(1.0,1.4,2.3,3.1) \times 10^{-5}$, and $v_{3}^{H}=(2.3,3.1,3.5,3.5) \times 10^{-5}$, respectively. The single-valued input is $2.5 \times 10^{-5}$, which is presented by the vertical purple line. The output of each category is the cross point between the corresponding MF curve and the purple line. In this case, the outputs for 'low', 'medium', and 'high' are $0,0.75$, and 0.25 , respectively. 


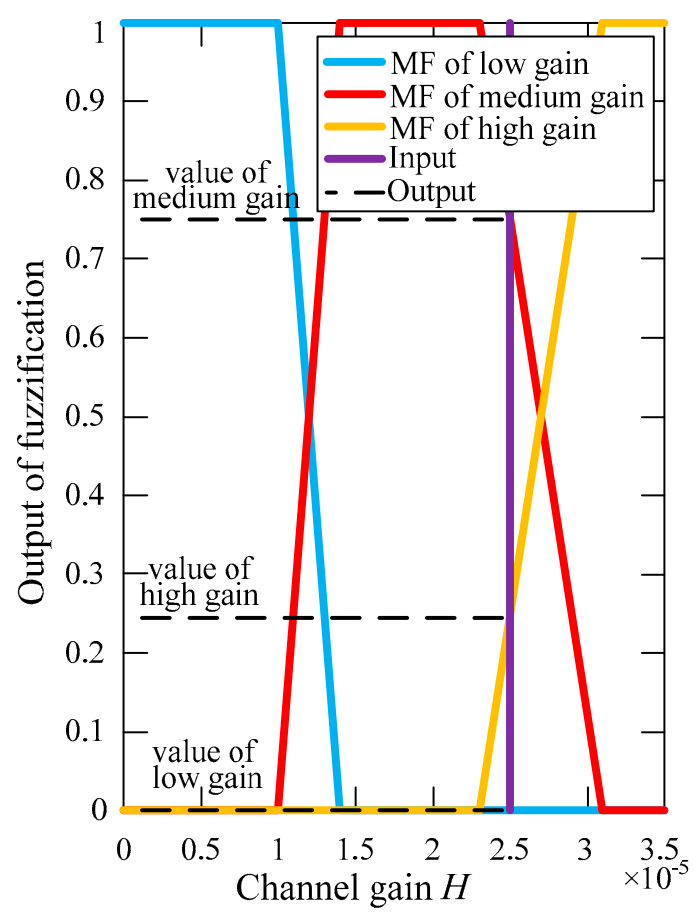

Figure 3. Fuzzification process of channel gain.

- Rule evaluation

The user priority for bandwidth allocation can be classified into several states. The number of states is denoted by $n_{4}$. When $n_{4}=3$, the states can be 'negative', 'neutral', and 'positive'. The relationship between the states and the input features is clarified by fuzzy rules. When $n_{1}=4$, $n_{2}=3, n_{3}=2$, and $n_{4}=3$, the fuzzy rules are shown in Table 1 . The output of each rule is the minimum value of three involved components and the final value for each state is the maximum output of the rules with the same state. The final value of 'negative', 'neutral', and 'positive' are denoted by $v_{l}$, $v_{m}$, and $v_{h}$, respectively. The fuzzy rules are clear. For example, when the communication condition is perfect, it is beneficial to allocate more resource to users (rule 1 and rule 2); when the communication condition is poor, it is better to allocate resource to users (rule 10).

Table 1. Fuzzy rules.

\begin{tabular}{ccccc}
\hline No. & $\boldsymbol{R}$ & $\boldsymbol{H}$ & $\boldsymbol{p}$ & State \\
\hline 1 & - & High & Low & Positive \\
2 & High & Med & Low & Positive \\
3 & High or Med-High & High & High & Neutral \\
4 & Not High & Med & Low & Neutral \\
5 & High & Med & High & Neutral \\
6 & High or Med-High & Low & Low & Neutral \\
7 & Low-Med or Low & High & High & Negative \\
8 & Not High & Med & High & Negative \\
9 & Low-Med or Low & Low & Low & Negative \\
10 & - & Low & High & Negative \\
\hline
\end{tabular}

- Defuzzification

This step obtains a priority score of each user for bandwidth allocation. The MFs which are used to describe the relation between the priority score $s$ and the aforementioned states in Table 1 are expressed as

$$
F_{s}\left(n_{4}\right)=\left\{f_{i}^{s}\left(s ; v_{i}^{s}=\left(a_{i}, b_{i}, c_{i}, d_{i}\right)\right) \mid i=1, \cdots, n_{4}\right\} .
$$


Equation (10) means that the priority score $s$ is classified into $n_{4}$ categories. Each category is a state in Table 1. The membership degrees to different states are obtained from the rule evaluation process by Table 1. The defuzzification process utilizes the membership degrees to different states to calculate the priority score. The centroid method is widely adopted in the defuzzification process for the calculation of the priority score. When $n_{4}=3, v_{1}^{s}=(0,0,0.2,0.4), v_{2}^{s}=(0.2,0.5,0.5,0.8), v_{3}^{s}=(0.6,0.8,1,1)$, $v_{l}=0.1, v_{m}=0.6$, and $v_{h}=0.3$, the defuzzification process using the centroid method is shown in Figure 4. For each state, the area that is below both its final state value and its MF is shaded in black in the figure. Taking state 'negative' as an example, the shaded area is below both the blue MF and the horizontal line marked 'value of negative'. The three shaded areas of the three states merge into a whole shaded area and the upper edge of the whole shaded area is denoted by $f(x)$. Obviously, $f(x)$ is a piecewise curve. Using the centroid method, the priority score of each user is calculated by [14]

$$
s=\frac{\int_{0}^{1} f(x) x d x}{\int_{0}^{1} f(x) d x}
$$

where the priority score ranges from 0 to 1 . Actually, the centroid method is a weighting method for the final values of states. In Figure 4, the priority output through the defuzzification process is 0.56 .

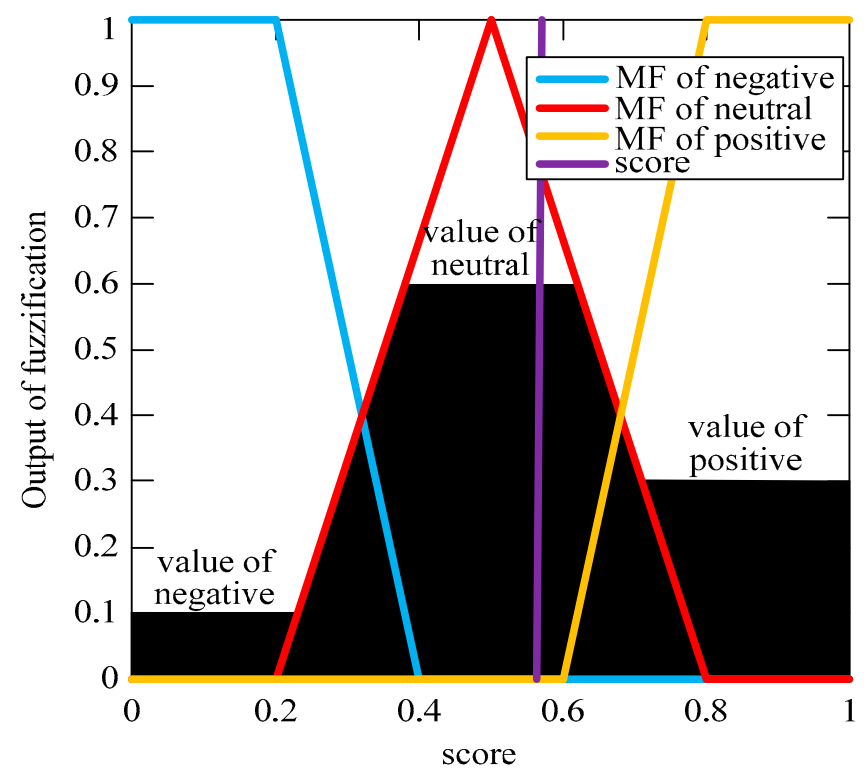

Figure 4. Defuzzification using the centroid method.

\subsection{Bandwidth Allocation}

The user priority vector obtained from the priority measurement model is denoted by $\vec{s}=\left\{s_{1} \cdots, s_{k}, \cdots, s_{N}\right\}$. By sorting $\vec{s}$ in descending order, we get another user priority vector $\overrightarrow{s^{\prime}}=\left\{s_{1}^{\prime} \cdots, s_{k^{\prime}}^{\prime} \cdots, s_{N}^{\prime}\right\}$. We can refer to the corresponding element of vector $\vec{s}$ from vector $\overrightarrow{s^{\prime}}$ by 
index vector index $=\left\{\right.$ index $_{1}, \cdots$, index $x_{k}, \cdots$, index $\left._{N}\right\}$, which means $s_{k}=s_{\text {index }}^{\prime}$. The proposed TMRA scheme is expressed as

$$
\begin{aligned}
& \max C=\sum_{k=1}^{N} C_{k} \\
& \text { s.t. }\left\{\begin{array}{l}
\sum_{k=1}^{N} B_{k}=B, 1 \leq k \leq N \quad(12 b) \\
B_{k} \geq B_{j}, \forall \text { index }_{k} \leq \text { index } \\
\\
B_{k} \geq 0,1 \leq k, j \leq N
\end{array}\right.
\end{aligned}
$$

where the objective is to maximize the system throughput $C ;(12 \mathrm{~b})$ indicates the total bandwidth of the system is $B ;(12 \mathrm{c})$ means to provide priority guarantee. The proposed allocation method based on DUP consists of the DUP model and the TMRA scheme. It is denoted as DUP-TMRA method.

When (12c) becomes $B_{k}=B_{j}$, we obtain the allocation method called equal proportion allocation (EPA) method. The EPA is a special case of the allocation method space for (12). Therefore, the throughput of EPA is a lower bound of the proposed DUP-TMRA method. The lower bound is denoted by $E[C]$ where $E[\cdot]$ represents the expectation of $C$. For the $k$-th user, random variable $H_{k}$ is a function of position random variables $x_{k}$ and $y_{k}$. It is assumed that $x_{k}, y_{k}$, and are mutually independent. The system throughput $C$ is the sum of the throughput of all users numbered from 1 to $N$ in the networks. The derivation process of the lower bound is based on the EPA method.

$$
\begin{aligned}
E[C] & =\sum_{k=1}^{N} E\left[C_{k}\right] \\
& =\sum_{k=1}^{N} E\left[\left(1-p_{k}\right) \frac{B}{N} \log _{2}\left(1+\frac{\left(r H_{k} \frac{P_{t}}{N}\right)^{2}}{n_{0} \frac{B}{N}}\right)\right] \\
& =\frac{B}{N} \sum_{k=1}^{N} E\left[1-p_{k}\right] E\left[\log _{2}\left(1+\frac{\left(r H_{k} P_{t}\right)^{2}}{n_{0} B N}\right)\right]
\end{aligned}
$$

where $P_{t}$ is the power of the AP; the bandwidth allocated to each user is $B / N$; the power allocated to each user is $P_{t} / N$. In (13), $E\left[1-p_{k}\right]=1-\bar{p}$. With $\rho=\frac{\left(r P_{t}(m+1) h^{m+1} A T_{S}(\psi) n^{2}\right)^{2}}{n_{0} B\left(2 \pi \sin ^{2} \Psi_{c}\right)^{2}}$, we have

$$
\begin{aligned}
& E\left[\log _{2}\left(1+\frac{\left(r H_{k} P_{t}\right)^{2}}{n_{0} B N}\right)\right] \\
& =E\left[\log _{2}\left(1+\frac{\rho}{N\left(x_{k}^{2}+y_{k}^{2}+h^{2}\right)^{m+3}}\right)\right] \\
& =\frac{1}{S_{0} \ln 2} \iint_{S} \ln \left(1+\frac{\rho}{N\left(x_{k}^{2}+y_{k}^{2}+h^{2}\right)^{m+3}}\right) d x_{k} d y_{k}
\end{aligned}
$$

where $S_{0}$ is the area of the room; $S$ is the integral region. When $N$ is less than 10, $\rho /\left(N\left(x_{k}^{2}+y_{k}^{2}+h^{2}\right)^{m+3}\right)$ is much larger than 1 . Therefore, an approximate treatment for (14) is expressed as

$$
\begin{aligned}
& E\left[\log _{2}\left(1+\frac{\left(r H_{k} P_{t}\right)^{2}}{n_{0} B N}\right)\right] \\
\approx & \frac{1}{S_{0} \ln 2} \iint_{S} \ln \left(\frac{\rho}{N\left(x_{k}^{2}+y_{k}^{2}+h^{2}\right)^{m+3}}\right) d x_{k} d y_{k} \\
= & \frac{1}{S_{0} \ln 2} \iint_{S} \ln \left(\frac{\rho}{\left(x_{k}^{2}+y_{k}^{2}+h^{2}\right)^{m+3}}\right) d x_{k} d y_{k} \\
& \quad-\frac{1}{S_{0} \ln 2} \iint_{S} \ln N d x_{k} d y_{k} \\
= & b_{0}-\log _{2} N
\end{aligned}
$$


where $b_{0}$ is independent of $N$. Substituting (14) and $E\left[1-p_{k}\right]=1-\bar{p}$ into (13), the lower bound of the proposed DUP-TMRA method is expressed as

$$
\begin{gathered}
E[C] \approx \frac{B}{N} \sum_{k=1}^{N}(1-\bar{p})\left(b_{0}-\log _{2} N\right) \\
=B(1-\bar{p})\left(b_{0}-\log _{2} N\right)
\end{gathered}
$$

Equation (16) indicates that the lower bound is related to system bandwidth, average blocking probability and the total number of users. The increase of the number of users would cause the reduction of the system throughput.

\section{Simulation Results}

In this section, the Monte Carlo simulations are used to evaluate the performance of the proposed method. A single-cell scene with a squared room and an AP is considered. The vertical projection of the AP is exactly in the center of the ceiling. According to [14,15], we choose the dynamic user priority proportion allocation (DUP-PA) method, the EPA method and the RPA method as comparison methods, and they are also denoted as DUP-PA, E-PA, and R-PA, respectively. We choose system throughput, the degree of satisfaction of user, and the fairness among users as key performance indicators (KPIs). The system throughput is expressed as

$$
C=\sum_{k=1}^{N} C_{k}
$$

The degree of satisfaction of the $k$-th user is given by [14]

$$
\chi_{k}= \begin{cases}C_{k} / R_{k}, & C_{k}<R_{k} \\ 1, & C_{k} \geq R_{k}\end{cases}
$$

The user whose degree of satisfaction equals to 1 is defined as a satisfied user. The fairness among users is measured by Jain's fairness index [14], which is calculated by

$$
\xi=\frac{\left(\sum_{k=1}^{N} \chi_{k}\right)^{2}}{N \sum_{k=1}^{N} \chi_{k}^{2}}
$$

\subsection{Condition}

Unless specifically stated, simulation parameters are shown in Table 2. The set of MFs of channel gain $H$, the set of MFs for defuzzification and fuzzy rules table are shown in Figure 3, Figure 4 and Table 1, respectively. The set of MFs of required data rate $R$ is expressed as [20]

$$
F_{R}(4)=\left\{\begin{array}{l}
f_{1}^{R}\left(R ; v_{1}^{R}=\left(0,0,0, \frac{3}{4} \bar{R}\right)\right), f_{2}^{R}\left(R ; v_{2}^{R}=\left(\frac{1}{2} \bar{R}, \bar{R}, \bar{R}, \frac{3}{2} \bar{R}\right)\right), \\
f_{3}^{R}\left(R ; v_{3}^{R}=\left(\frac{5}{4} \bar{R}, \frac{7}{4} \bar{R}, \frac{7}{4} \bar{R}, \frac{9}{4} \bar{R}\right)\right), f_{4}^{R}\left(R ; v_{4}^{R}=(2 \bar{R}, 4 \bar{R}, 4 \bar{R}, 4 \bar{R})\right)
\end{array}\right\}
$$

and the set of MFs of blocking probability $p$ is given by

$$
F_{p}(2)=\left\{f_{1}^{p}\left(p ; v_{1}^{p}=\left(0,0,0, \frac{3}{4} \bar{p}\right)\right), f_{2}^{p}\left(p ; v_{2}^{p}=\left(\frac{1}{2} \bar{R}, \bar{R}, \bar{R}, \frac{3}{2} \bar{p}\right)\right)\right\}
$$


Table 2. Simulation parameters.

\begin{tabular}{cc}
\hline Parameter & Value \\
\hline Room size & $2.5 \times 2.5 \times 3 \mathrm{~m}$ \\
Transmit optical power of AP, $P_{t}$ & $9 \mathrm{~W}$ \\
Height between the AP and user, $h$ & $1.65 \mathrm{~m}$ \\
Half-intensity radiation angle, $\Phi_{1 / 2}$ & $60^{\circ}$ \\
FOV semi-angle of PD, $\Psi_{c}$ & $60^{\circ}$ \\
The refractive index, $n$ & 1.5 \\
Detector responsivity, $r$ & $0.53 \mathrm{~A} / \mathrm{W}$ \\
The physical area of a PD, $A$ & $10^{-4} \mathrm{~m}^{2}$ \\
Power spectral density of noise, $n_{0}$ & $10^{-21} \mathrm{~A}^{2} / \mathrm{Hz}$ \\
Bandwidth per optical AP, $B$ & $40 \mathrm{MHz}$ \\
The gain of the optical filter, $T_{S}(\psi)$ & 1 \\
Average required data rate, $\bar{R}$ & $40 \mathrm{Mbps}$ \\
Average blocking probability, $\bar{p}$ & 0.1 \\
The number of users, $N$ & 10 \\
\hline
\end{tabular}

\subsection{Dynamic User Priority Model Analysis}

\subsubsection{Analysis on Feature Dimension}

We study the advantage of the proposed three-dimensional DUP model under different user density levels. For resource allocation, the conventional proportion allocation scheme is adopted uniformly. We choose the dynamic one-dimensional priority model and the fixed priority model as comparison models. The allocation method adopting the proposed DUP model is denoted as DUP-PA. The comparison methods, in which the corresponding one-dimensional feature is required data rate, channel gain, and blocking probability are denoted as R-PA, CG-PA, and RP-PA, respectively. We choose the E-PA method as an allocation method with fixed user priority. This is because when the number of users is fixed, the priority of users for the E-PA method will not change with the dynamic network environment. The result is in Figure 5.

According to Figure 5, the system throughput decreases for all methods as $N$ increases, which means $N$ has a universal effect on system throughput. When $N$ is fixed, the DUP-PA method outperforms R-PA, CG-PA, and RP-PA, and the throughput improvement ratios range from 1.7\% to $3.3 \%, 1.0 \%$ to $1.4 \%$, and $0.3 \%$ to $3.9 \%$, respectively. It means that feature dimension has a great effect on the performance of the system and the high-dimensional priority model outperforms the low-dimensional priority model. In addition, the improvement ratios become more significant when $N$ increases, which means the proposed DUP model is more suitable for high user density scenes. The DUP-PA method outperforms the E-PA method, which indicates that the proposed DUP model outperforms the conventional fixed user priority model.

The reason why the DUP-PA method outperforms the comparison methods is that the DUP-PA method has a more comprehensive consideration on network environment. When $N$ increases, RP-PA has the highest descending rate in system throughput, which means that the RP-PA is the most sensitive to user density. Because the CG-PA outperforms the R-PA method and it begins to outperform the RP-PA when $N$ is larger than 4 , the channel gain is the central feature among the selected three features.

In Figure 6, the fairness among users is shown as a function of $N$. The fairness among users decreases as $N$ increases which indicates that it will be more difficult to guarantee fairness when the user density is higher. The DUP-PA method is inferior to the R-PA method and the CG-PA method, but it outperforms the RP-PA method a lot. When $N$ is as large as 10, the fairness among users of the DUP-PA method can still maintains as high as 0.95 , which indicates that the proposed DUP model does not significantly reduce the fairness among users. Therefore, the DUP-PA method still performs well in terms of fairness. According to Figures 5 and 6, we have the conclusion that the proposed DUP model can produce considerable throughput growth without sacrificing the fairness among users significantly. 


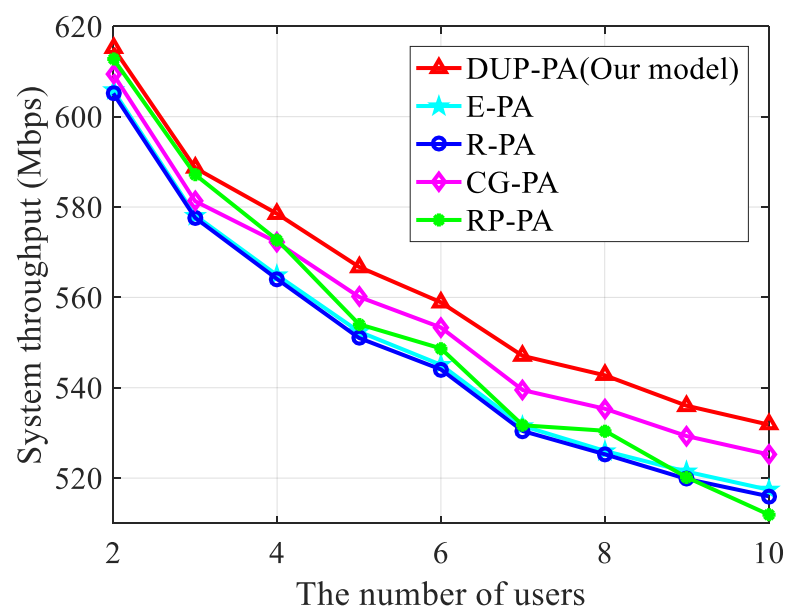

Figure 5. Feature analysis for the proposed DUP model.

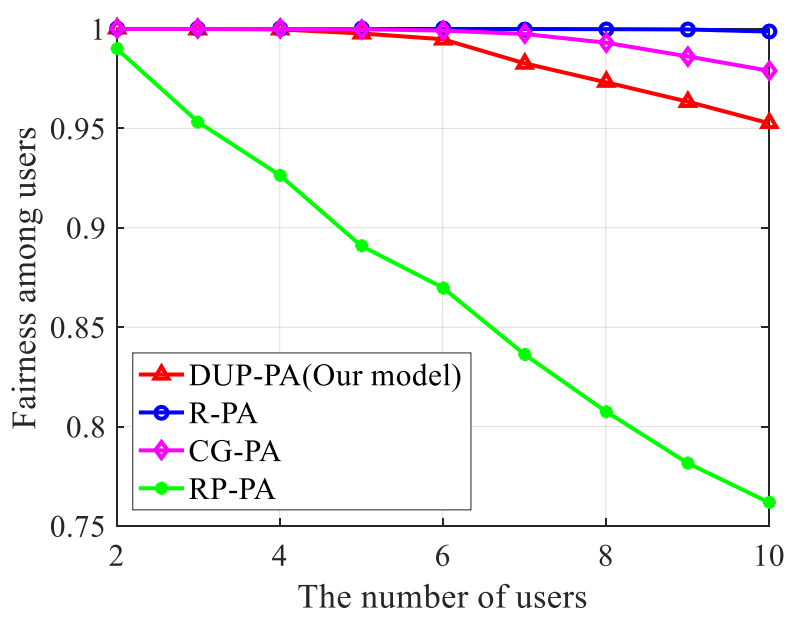

Figure 6. The effect of features on the fairness among users.

\subsubsection{Analysis on Average Required Data Rate}

In this subsection, we study the effect of user's average required data rate $\bar{R}$ and the result is shown in Figure 7. The method, in which the one-dimensional feature is required rate and the allocation scheme is the proposed TMRA scheme, is denoted as R-TMRA method. According to Figure 7, the proposed DUP-TMRA method outperforms the R-TMRA, E-PA, and R-PA. The system throughput of each method will converge to a stable value, which indicates that the system throughput will not grow indefinitely as user's requirement increases. This result is consistent with the limitation of bandwidth resource.

The R-PA method is more sensitive to $\bar{R}$. When $\bar{R}$ is less than $10 \mathrm{Mbps}$, the system throughput of R-PA grows rapidly; when $\bar{R}$ is greater than $10 \mathrm{Mbps}$, the throughput grows slowly at first, and finally converges to a certain value. The R-TMRA method is not sensitive to $\bar{R}$ and it outperforms the R-PA, which means that when the same user priority measurement model is adopted, the proposed TMRA scheme with priority guarantee will make the system throughput stable. Similarly, the reason why the proposed method is not sensitive to $\bar{R}$ can be explained. In (16), the lower bound of system throughput is not the function of $\bar{R}$, which can explain the reason why the E-PA method is not sensitive to $\bar{R}$. Therefore, the theoretical analysis in Section 3.3 is consistent with the simulation result in Figure 7. According to Figure 7, when user's requirement changes greatly, the proposed method can still maintain a stable throughput, which means that the system has high stability under the proposed method. 


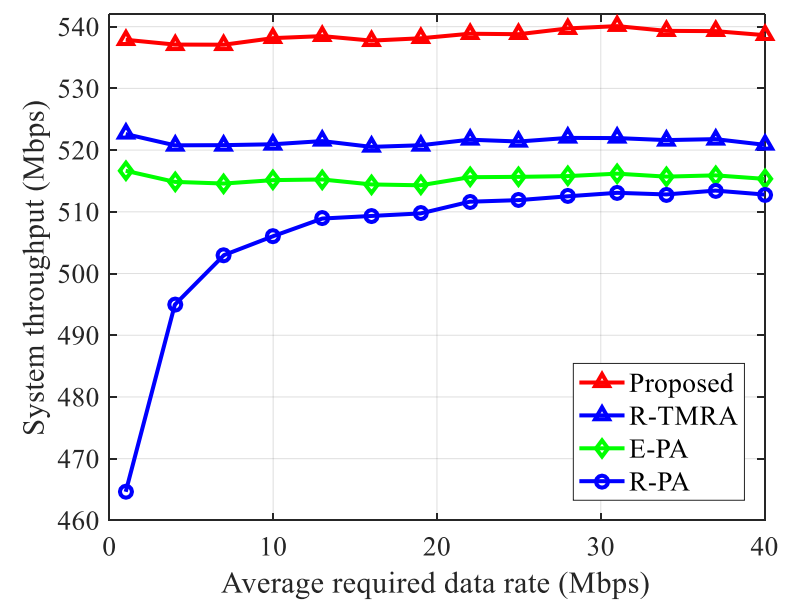

Figure 7. System throughput versus average required data rate.

Figure 8 shows the system throughput improvement ratios of the proposed method compared to the R-TMRA, E-PA, and R-PA, which range from $2.9 \%$ to $3.5 \%, 4.1 \%$ to $4.6 \%$, and $5.0 \%$ to $15.8 \%$, respectively. When user's average required rate is high enough, the improvement ratio will converge to a stable value.

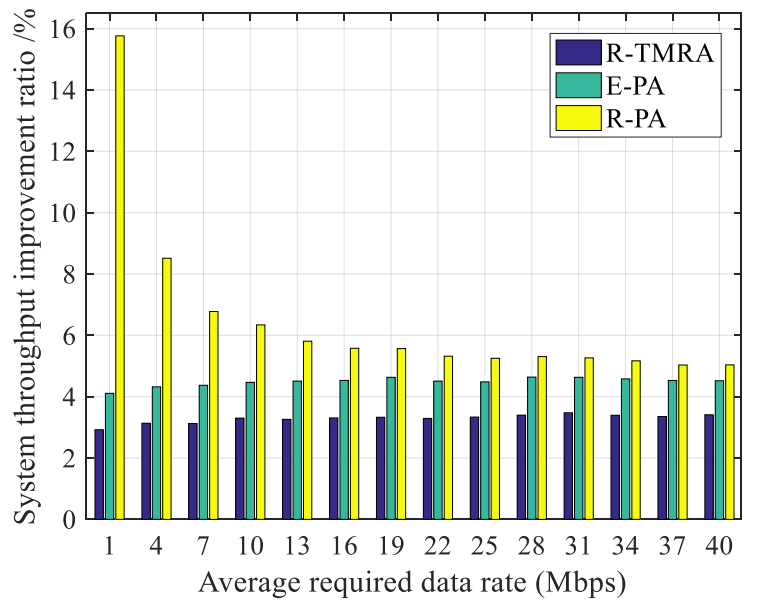

Figure 8. System throughput improvement ratio versus average required data rate.

\subsubsection{Analysis on Average Blocking Probability}

In this subsection, we study the effect of blocking and the result is shown in Figure 9. According to Figure 9, when user's average blocking probability $\bar{p}$ is fixed, the proposed method outperforms the three comparison methods. The performance curves of the four methods have the same trend. The system throughput is an approximate negative linear function of $\bar{p}$, which indicates that the simulation result in Figure 9 is consistent with the theoretical analysis of (16) in Section 3.3. The proposed method slows down the decline of system throughput. For example, when $\bar{p}$ is 0.15 , the throughput of the proposed method only decreases by $12.8 \%$. According to (16), the lower bound of system throughput is a negative linear function of $\bar{p}$, and it is a negative logarithmic function of $N$, which indicates that blocking probability has a greater impact on throughput than the number of users. 


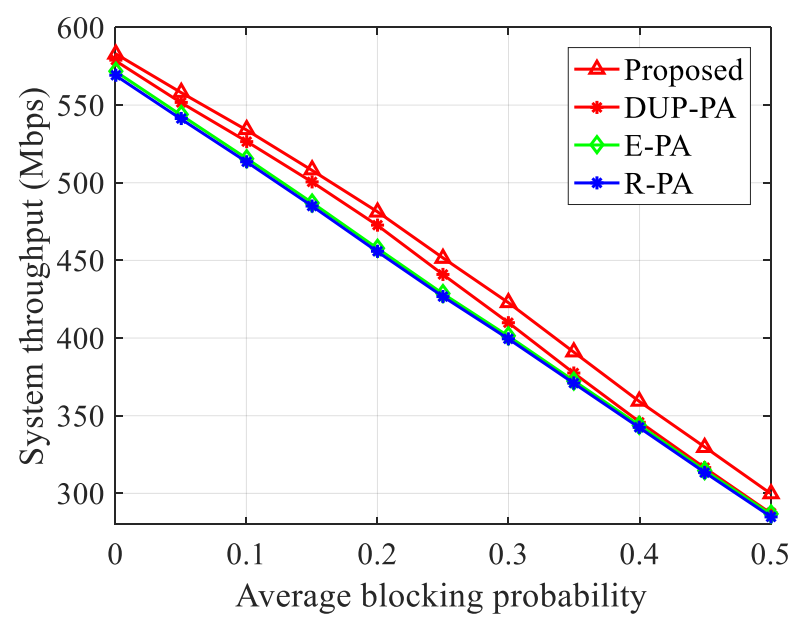

Figure 9. System throughput versus average blocking probability.

According to Figure 10, the system improvement ratios compared with the E-PA and the R-PA follow a single peak curve, and when $\bar{p}$ is 0.25 , the highest performance improvement is obtained. Compared with DUP-PA, the system improvement ratio increases as $\bar{p}$ increases. When $\bar{p}$ ranges from 0 to 0.5 , the performance improvement ratios, compared with the DUP-PA, the E-PA, and the R-PA, range from $0.8 \%$ to $4.4 \%, 2.0 \%$ to $5.4 \%$, and $2.5 \%$ to $5.8 \%$, respectively. The simulation result indicates that the proposed method is more tolerant to channel blocking and it is more suitable for scenes with high user density.

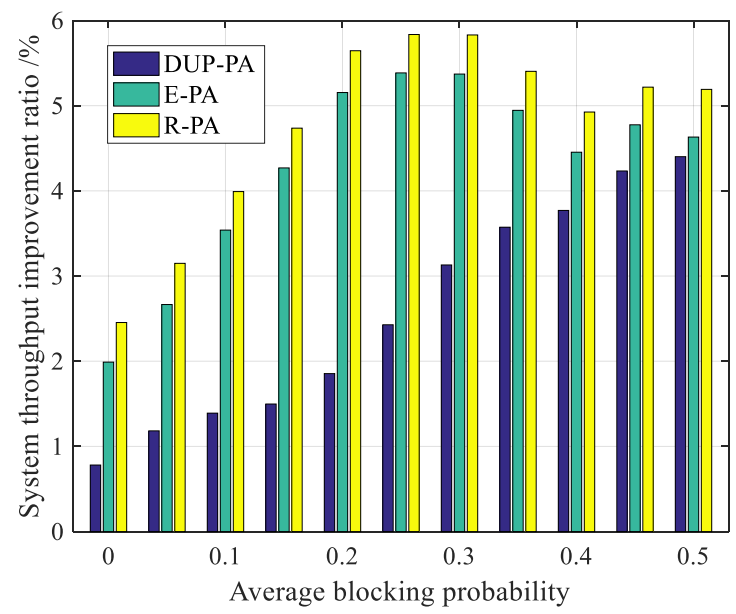

Figure 10. System throughput improvement ratio versus average blocking probability.

\subsubsection{Analysis on Channel Gain}

In this subsection, we study the effect of channel gain. According to (1), there are several parameters influence the channel gain of a user. Among these parameters, FOV is a critical scene parameter in VLC-UDNs and it is expected to significantly influence the performance of networks. Therefore, we study the effect of FOV and the result is shown in Figure 11. In general, the system throughput decreases as user's FOV increases. This is because that the increase in FOV causes a decrease in $g\left(\psi_{k}\right)$ and $H_{k}$ according to (1) and (2). This indicates that when the overall level of the channel gain is higher, the performance of the system is better. According to Figures 5 and 11, the R-PA method has a similar performance with the E-PA method and the increase of FOV cannot enhance the gap between the E-PA method and the R-PA method. When FOV ranges from $50^{\circ}$ to $80^{\circ}$, the performance improvement ratios, compared with the DUP-PA, E-PA, and R-PA, approximate to $1.7 \%$, $4.5 \%$, and $4.8 \%$, respectively. 


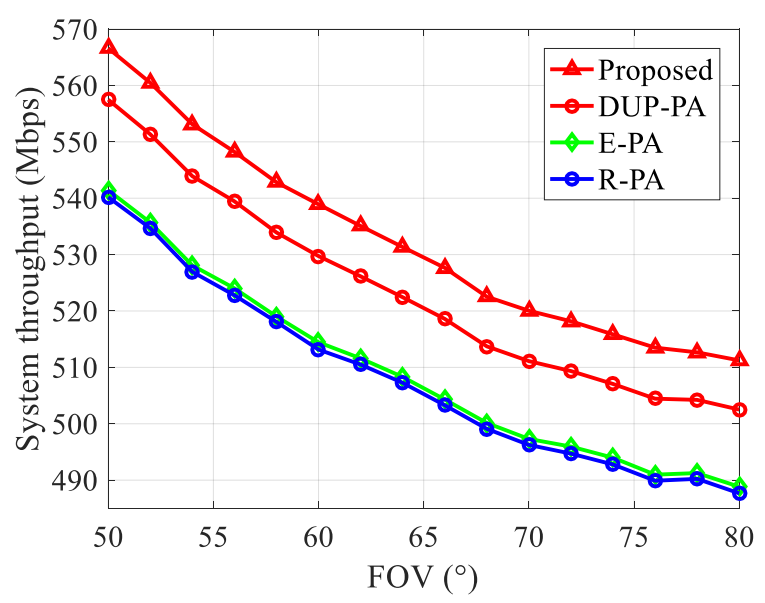

Figure 11. System throughput versus the field of view of users.

\subsection{Performance Analysis for the Proposed DUP-TMRA Method}

\subsubsection{Comparisons in Allocation Schemes}

In this subsection, we analyze the advantage of the proposed TMRA scheme compared with the conventional proportion allocation scheme. The method, in which the one-dimensional feature is channel gain and the allocation scheme is the proposed TMRA scheme, is denoted as CG-TMRA method. The result is shown in Figure 12. The R-TMRA method outperforms the R-PA method and the performance improvement ratio ranges from $1.28 \%$ to $1.77 \%$. The CG-TMRA method outperforms the CG-PA method and the performance improvement ratio ranges from $1.21 \%$ to $2.36 \%$. It means that the proposed TMRA scheme has a better performance than the conventional proportion allocation scheme.

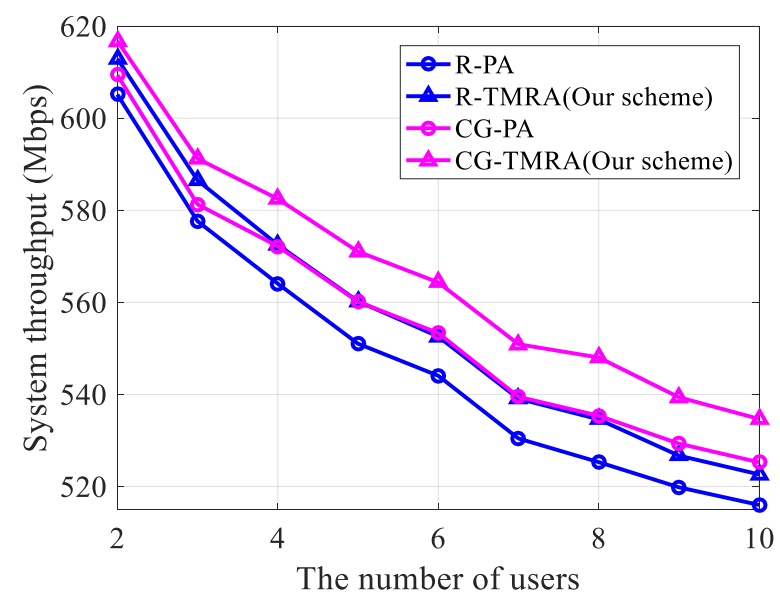

Figure 12. Comparison between the TMRA scheme and the conventional proportion allocation scheme.

\subsubsection{Analysis on System Throughput}

In this subsection, we study the effect of user density level on system throughput as shown in Figure 13. The trend of the four curves is roughly the same. The system throughput decreases as $N$ increases, which means that $N$ has a universal impact on system throughput and high user density will cause low bandwidth utilization efficiency. According to (16), the first derivative of the lower bound versus the number of users $N$ is always less than 0 , and the second derivative of the lower bound versus the number of users $N$ is always larger than 0 , which explains the reason for the trend of the curves. This indicates that for the effect of the number of users on system throughput, the theoretical analysis in Section 3.3 is consistent with the simulation result in Figure 13. 


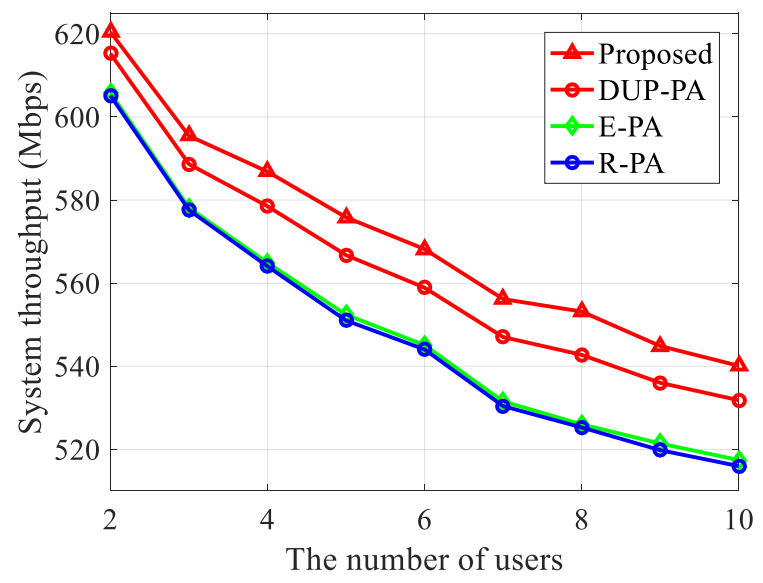

Figure 13. System throughput versus the number of users.

The proposed method performs better than the DUP-PA method, this is because that the two methods use the same priority measurement model, but the DUP-PA method is a special feasible method in the feasible set of the proposed method. The DUP-PA method outperforms the E-PA method and the R-PA method. This is because that in the process of priority measurement, the DUP-PA method takes into account three features of users and makes full use of user's information. The E-PA method does not use user's information effectively and the R-PA method only uses one-dimensional user information.

According to Figure 14, when $N$ is fixed, the proposed method outperforms the DUP-PA, the E-PA and the R-PA, and the throughput improvement ratios range from $0.9 \%$ to $1.9 \%, 2.4 \%$ to $5.2 \%$, and $2.5 \%$ to $5.3 \%$, respectively. In general, when $N$ is larger, the system throughput improvement ratio is higher. This indicates that the user density is higher, the advantage of the proposed method is greater and the proposed method is more suitable for high user density scenes.

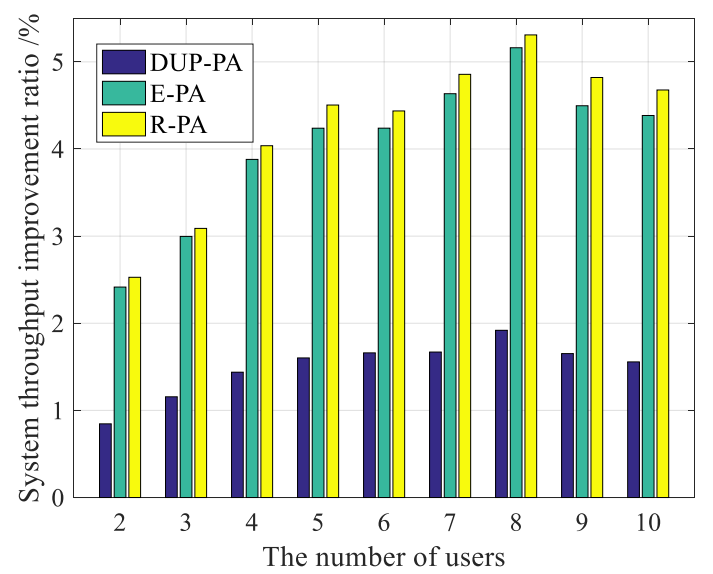

Figure 14. System throughput improvement ratio versus the number of users.

\subsubsection{Analysis on the Degree of Satisfaction}

In Figure 15, the proportion of the satisfied users is shown as a function of user's average blocking probability $\bar{p}$. The proportion of satisfied users decreases as $\bar{p}$ increases for the four curves, which indicates that when the communication condition becomes worse, the guarantee for quality of service (QoS) is reduced. 


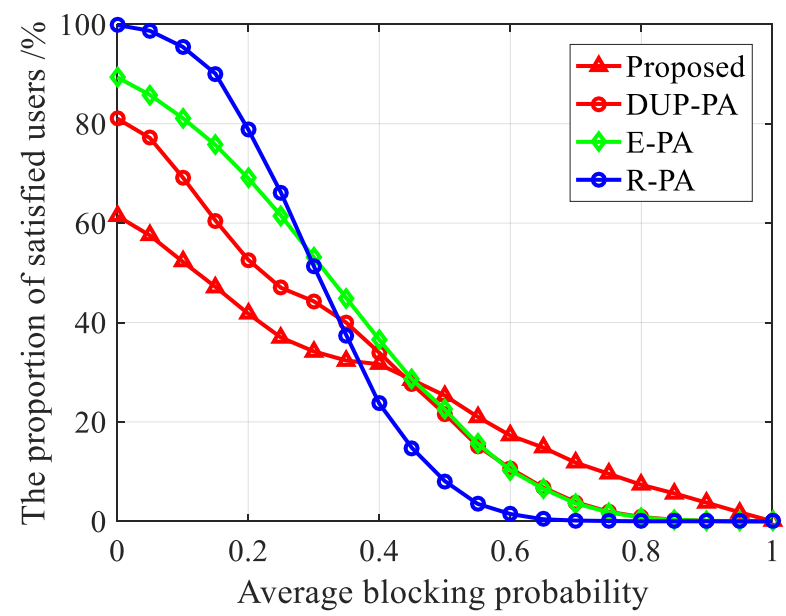

Figure 15. The proportion of satisfied users versus the average blocking probability.

When $\bar{p}$ is less than 0.45 , the performance of the proposed method is inferior to the comparison methods. This is because although the system throughput of the comparison methods is lower than the proposed method (Figure 9), the total throughput of comparison methods is still high. Furthermore, the comparison methods are more biased to meet the requirement of each user. Therefore, the performance of comparison methods can be better. In the contrast, when $\bar{p}$ is greater than 0.45 , the proposed method outperforms the comparison methods. This is because for all of the four methods, the system throughput decreases as $\bar{p}$ increases (Figure 9), and when the required data rate is fixed, the advantage of the proposed method on system throughput outweighs the disadvantage on not being biased to meet the requirement of users.

When $\bar{p}$ increases to 0.8 , the proportion of satisfied users of three comparison methods almost equals to zero, but the proportion of satisfied users of the proposed method can still be as high as $7.13 \%$. In addition, when $\bar{p}$ equals to 0.55 , the proposed method has the maximum performance improvement ratio compared with the conventional R-PA method and the improvement ratio is $17.5 \%$ In conclusion, the proposed method not only has the advantage of throughput (Figure 9), but also has the advantage of the degree of satisfaction in high blocking condition (Figure 15).

When $\bar{p}=0.55$, the distribution detail of the degree of satisfaction is shown in Figure 16. The proposed method can meet the data rate requirements for $21.01 \%$ of the users. The proportion of satisfied users for the DUP-PA, E-PA, R-PA is $15.17 \%, 15.65 \%$, and $3.52 \%$, respectively. Note that there are cross points between the curves of the proposed method and the comparison methods. This is because the proposed method can improve the proportion of deeply-satisfied users. When the degree of satisfaction is smaller than a certain value, the CDF curve of the proposed method is above the curves of the comparison methods, which indicates that the proportion of deeply-unsatisfied users for the proposed method is higher. Because the proportion of deeply-satisfied users and deeply-unsatisfied users for the proposed method is higher than comparison method, the proportion of users with middle-level degree of satisfaction is lower. 


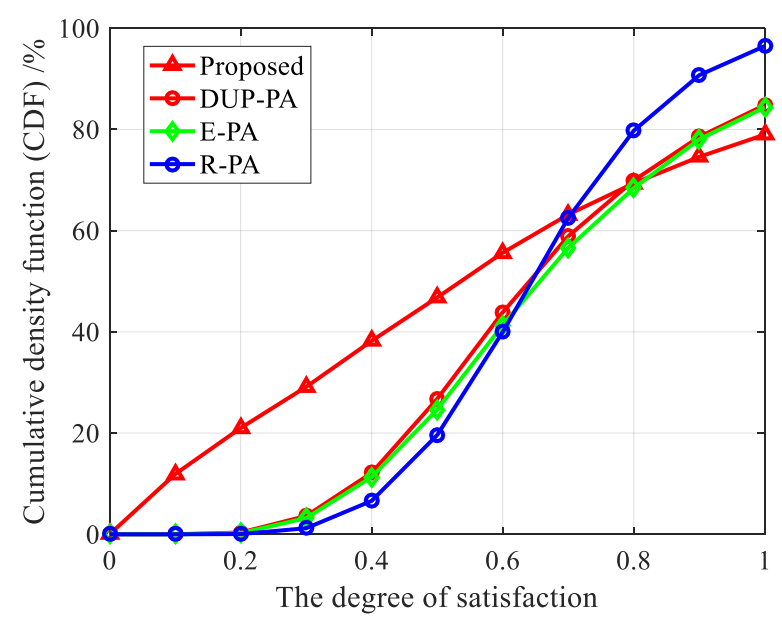

Figure 16. The cumulative density function $(\mathrm{CDF})$ versus the degree of satisfaction $(\bar{p}=0.55)$.

\section{Conclusions}

In this paper, we proposed the resource allocation method based on DUP named DUP-TMRA method for indoor VLC-UDNs. First, we established the DUP model with three features using FL method. Then, the TMRA scheme with priority guarantee was proposed. Simulation results proved that the proposed DUP model outperforms the conventional fixed priority model and dynamic priority model with one feature. Channel gain is the central feature of the proposed DUP model. The proposed TMRA scheme shows a better performance than the conventional proportion allocation scheme. Blocking probability has a greater impact on throughput than the number of users. Because the RPA method has a similar performance with the EPA method, the system throughput of the EPA method can be regarded as the lower bound of the proposed DUP-TMRA method. The proposed method improves the system throughput against the conventional RPA method by $4 \%$. In addition, when the average blocking probability is higher than 0.45 , the proposed method has an advantage on the degree of satisfaction, and it improves the proportion of satisfied users by up to $17.5 \%$. The proposed method is suitable for VLC-UDNs with high user density. Future research will study the adaptive updating of fuzzy logic rule tables and resource allocation in multi-cell VLC-UDNs.

Author Contributions: X.-W.B., Q.L. and S.-Y.T. contributed to conceptualization; X.-W.B. and S.-Y.T. contributed to develop the methodology; X.-W.B. contributed to software; X.-W.B. contributed to investigation; X.-W.B. contributed to original draft preparation; X.-W.B., Q.L. and S.-Y.T. contributed to revision work.

Funding: This research was funded by National Natural Science Foundation of China (grant no. 61671477).

Conflicts of Interest: The authors declare no conflict of interest.

\section{References}

1. Haas, H.; Yin, L.; Wang, Y.; Chen, C. What is LiFi? J. Lightwave Technol. 2016, 34, 1533-1544. [CrossRef]

2. Feng, L.; Hu, R.Q.; Wang, J.; Xu, P.; Qian, Y. Applying VLC in 5G Networks: Architectures and Key Technologies. IEEE Network 2016, 30, 77-83. [CrossRef]

3. Bawazir, S.S.; Sofotasios, P.C.; Muhaidat, S.; Al-Hammadi, Y.; Karagiannidis, G.K. Multiple Access for Visible Light Communications: Research Challenges and Future Trends. IEEE Access 2018, 6, 26167-26174. [CrossRef]

4. Chen, S.; Qin, F.; Hu, P.D.B.; Li, X.; Liu, J. User-Centric Ultra-Dense Networks for 5G; Springer Nature: Basingstoke, UK, 2018; p. 7.

5. Kafafy, M.; Fahmy, Y.; Abdallah, M.; Khairy, M. Power Efficient Downlink Resource Allocation for Hybrid RF/VLC Wireless Networks. In Proceedings of the 2017 IEEE Wireless Communications and Networking Conference (WCNC), San Francisco, CA, USA, 19-22 March 2017; pp. 1-6. 
6. Wu, W.; Zhou, F.; Yang, Q. Dynamic network resource optimization in hybrid VLC and radio frequency networks. In Proceedings of the 2017 International Conference on Selected Topics in Mobile and Wireless Networking (MoWNeT), Avignon, France, 17-19 May 2017; pp. 1-7.

7. Dong, X.; Chi, X.; Sun, H.; Zhu, Y. Scheduling with heterogeneous qos provisioning for indoor visible-light communication. Curr. Opt. Photonics 2018, 2, 39-46.

8. Uddin, M.S.; Chowdhury, M.Z.; Jang, Y.M. Priority-based resource allocation scheme for visible light communication. In Proceedings of the 2010 Second International Conference on Ubiquitous and Future Networks (ICUFN), Jeju Island, Korea, 16-18 June 2010; pp. 247-250.

9. Chowdhury, M.Z.; Uddin, M.S.; Jang, Y.M. Dynamic channel allocation for QoS provisioning in visible light communication. In Proceedings of the 2011 IEEE International Conference on Consumer Electronics (ICCE), Las Vegas, NV, USA, 9-12 January 2011; pp. 13-14.

10. Chowdhury, M.Z.; Uddin, M.S.; Jang, Y.M. Dynamic Channel Allocation for Class-Based QoS Provisioning and Call Admission in Visible Light Communication. Arab. J. Sci. Eng. 2013, 39, 1007-1016. [CrossRef]

11. Chowdhury, M.Z.; Jang, Y.M.; Haas, Z.J. Priority based bandwidth adaptation for multi-class traffic in wireless networks. Int. J. Multimedia Ubiquitous Eng. 2014, 7, 445-450.

12. Wu, X.; Safari, M.; Haas, H. Three-state fuzzy logic method on resource allocation for small cell networks. In Proceedings of the 2015 IEEE 26th Annual International Symposium on Personal, Indoor, and Mobile Radio Communications (PIMRC), Hong Kong, China, 30 August-2 September 2015; pp. 1168-1172.

13. Wu, X.; Safari, M.; Haas, H. Bidirectional Allocation Game in Visible Light Communications. In Proceedings of the 2016 IEEE 83rd Vehicular Technology Conference (VTC Spring), Nanjing, China, 15-18 May 2016; pp. 1-5.

14. Wu, X.; Safari, M.; Haas, H. Access Point Selection for Hybrid Li-Fi and Wi-Fi Networks. IEEE Trans. Commun. 2017, 65, 5375-5385. [CrossRef]

15. Wang, Y.; Wu, X.; Haas, H. Fuzzy logic based dynamic handover scheme for indoor Li-Fi and RF hybrid network. In Proceedings of the 2016 IEEE International Conference on Communications (ICC), Kuala Lumpur, Malaysia, 22-27 May 2016; pp. 1-6.

16. Komine, T.; Nakagawa, M. Fundamental analysis for visible-light communication system using LED lights. IEEE Trans. Consum. Electron. 2004, 50, 100-107. [CrossRef]

17. Stefan, I.; Burchardt, H.; Haas, H. Area spectral efficiency performance comparison between vlc and rf femtocell networks. In Proceedings of the 2013 IEEE International Conference on Communications, Budapest, Hungary, 9-13 June 2013; pp. 3825-3829.

18. Jin, F.; Zhang, R.; Hanzo, L. Resource allocation under delay-guarantee constraints for heterogeneous visible-light and rf femtocell. IEEE Trans. Wireless Commun. 2015, 14, 1020-1034. [CrossRef]

19. Kashef, M.; Abdallah, M.; Qaraqe, K. Power allocation for downlink multi-user SC-FDMA visible light communication systems. In Proceedings of the 2015 49th Annual Conference on Information Sciences and Systems (CISS), Baltimore, MD, USA, 18-20 March 2015.

20. Burchardt, H.; Sinanovic, S.; Bharucha, Z.; Haas, H. Distributed and autonomous resource and power allocation for wireless networks. IEEE Trans. Commun. 2013, 61, 2758-2771. [CrossRef]

(C) 2019 by the authors. Licensee MDPI, Basel, Switzerland. This article is an open access article distributed under the terms and conditions of the Creative Commons Attribution (CC BY) license (http://creativecommons.org/licenses/by/4.0/). 